\title{
Critical care after the COVID-19 outbreak in China: lessons and renaissance
}

\author{
Jianfeng Xie ${ }^{1}$, Bin Du ${ }^{2^{*}}$ and Haibo Qiu ${ }^{1^{*}}$ (D)
}

(C) 2021 Springer-Verlag GmbH Germany, part of Springer Nature

Keywords: COVID-19, Critical care, Pandemic, Intensive care

Since the outbreak of the coronavirus disease 2019 (COVID-19) pandemic, more than 153 million cases have been confirmed, and more than 3.20 million individuals have died worldwide. The strategies of widespread testing, strict social distancing and isolation, information technology-based tracing, and widespread face mask use rapidly controlled the COVID-19 outbreak in China. Rapid responses and such strategies led to effective control in 1-2 months for several later localized outbreaks. On April 29th, there were only 324 confirmed cases in China; most of the cases were imported. Numerous lessons and experiences from this pandemic could impact and change critical care delivery in the future.

\section{Critical care resource reorganization}

During the first wave of the pandemic in Wuhan, a significant shortage of critical care resources led to an extreme crisis in fighting the pandemic. Approximately 3-4 thousand new cases were confirmed daily, among which $19 \%$ were severe and required intensive care [1]. However, there was a large gap between critical care delivery and demand. We only had 3.6 intensive care unit (ICU) beds per 100,000 capita compared with 34.7 in the US and 29.2

\footnotetext{
*Correspondence: dubin98@gmail.com; haiboq2000@163.com 1 Jiangsu Provincial Key Laboratory of Critical Care Medicine, Department of Critical Care Medicine, School of Medicine, Zhongda Hospital, Southeast University, Nanjing 210009, China

${ }^{2}$ State Key Laboratory of Complex, Severe and Rare Diseases, Medical ICU, Peking Union Medical College Hospital, Peking Union Medical College and Chinese Academy of Medical Sciences, Beijing 100730, China

Full author information is available at the end of the article
}

Haibo Qiu is the new Guest Editor 2021 for Intensive Care Medicine. in Germany, which made it difficult to meet the needs of a large number of critically ill patients during the pandemic [2]. The lack of professional critical care staff was another important shortage during the COVID-19 pandemic. We rapidly repurposed more than 9000 ward beds in 10 hospitals (including two urgent established hospitals) to critical care beds for critically ill patients in Wuhan. More than 42,000 medical staff, including $19,000(44.6 \%)$ critical care physicians and nurses, were recruited to Wuhan. The national critical care expert team went to Wuhan to guide medical management and infection control. These experiences were used to control later outbreaks in other cities.

To prepare for a future disaster similar to the COVID19 pandemic, the critical care system has been rebuilt and strengthened in China. Candidate hospitals were designed and rebuilt locally using special funds from the government. In each designated hospital, ICU beds represent $15-20 \%$ of total hospital beds or at least $200 \mathrm{ICU}$ beds. Each designated hospital also includes hundreds of beds that are suitable for both "low demand" and "high demand", which means that these beds can be used as wards and can be converted into ICU beds when needed. In addition, sufficient equipment (e.g., monitors, ventilators, continuous renal replacement therapy machines, extracorporeal membrane oxygenation and personal protective equipment) should be reserved in each designed hospital.

More importantly, increasing the number of welltrained and certified ICU doctors and nurses is crucial in China due to the considerable shortage. In designated critical care hospitals in Wuhan, the mortality for mechanically ventilated patients was 97\% [3]. Although

\section{实 Springer}


critical care medicine was recognized by the government as a primary specialty in China in 2008, no residency and fellowship training program for critical care medicine was available. A consensus on critical care training was generated in 2016, and a fellowship training program for critical care medicine was established and started in 2019. In April 2020, a residency training program was established for critical care medicine.

\section{Judicious use of antivirus and antibiotics}

Similar to other viral infections, COVID-19 is also a selflimited disease, and most patients are able to recover. Due to the rapidly growing number and to the high proportion of severe cases, the compassionate use of antivirals and antibiotics was very common during the early period of the pandemic. In our previous study, a majority of severe patients received combined antiviral treatment, such as arbidol, alpha interferon, hydroxychloroquine and lopinavir-ritonavir. Almost every patient received antibiotic treatment recommended by the first version of the surviving sepsis campaign (SSC) guidelines [4]. According to the results of clinical trials, the management of COVID-19 became much simpler in several late outbreaks in China (Fig. 1a). For example, no patient received combination therapy with antiviral agents, and only approximately $4 \%$ of all patients and $30 \%$ of critically ill patients received antibiotics in Xinjiang and Heilongjiang provinces.

\section{Clinical management}

There is no evidence for clinical application for such a new disease. Although the characteristics and mortality of COVID-19-induced acute respiratory distress syndrome (ARDS) are similar to those of typical ARDS [5], we also need to consider the physiopathology of the patients based on the guidelines due to the considerable heterogeneity of patients with ARDS. The treatment also needs to follow the principle of "one size does not fit all" and is based on the individual condition.

First, many patients have "silent hypoxemia" [6], which is difficult to recognize early and leads to delayed management of respiratory failure. Tobin MJ et al. explained the mechanism of silent hypoxemia in patients with COVID-19 [7]. Pulse oximetry-estimated saturation $\left(\mathrm{SpO}_{2}\right)$ was routinely monitored for every hospitalized patient. Patients who could get out of bed were asked to walk for $10 \mathrm{~m}$ under $\mathrm{SpO}_{2}$ monitoring, which we called the "oxygen stress test". If the $\mathrm{SpO}_{2}$ dropped below 93\% during or after walking, a high risk of developing severe conditions was noted. Unfortunately, the test is empirical. We do not have sufficient data to support this hypothesis, and further studies are encouraged. Additionally, an early warning system, including an oxygen stress test, lymphocyte count, and D-dimer levels, was established to identify severe patients.

Second, systemic hyperinflammation is not very common in severe COVID-19 patients [8]. Several studies have reported that cytokine storms play an important role during disease development in patients with COVID-19 [8]. Anti-inflammatory treatments, such as glucocorticoids or tocilizumab, were evaluated in clinical trials. The RECOVERY study showed that glucocorticoids could reduce mortality in patients with severe COVID-19 [9]. Approximately half of the patients received glucocorticoids in Wuhan; however, we found that glucocorticoid treatment was not associated with better outcomes in a cohort study [10]. We used an unsupervised machine

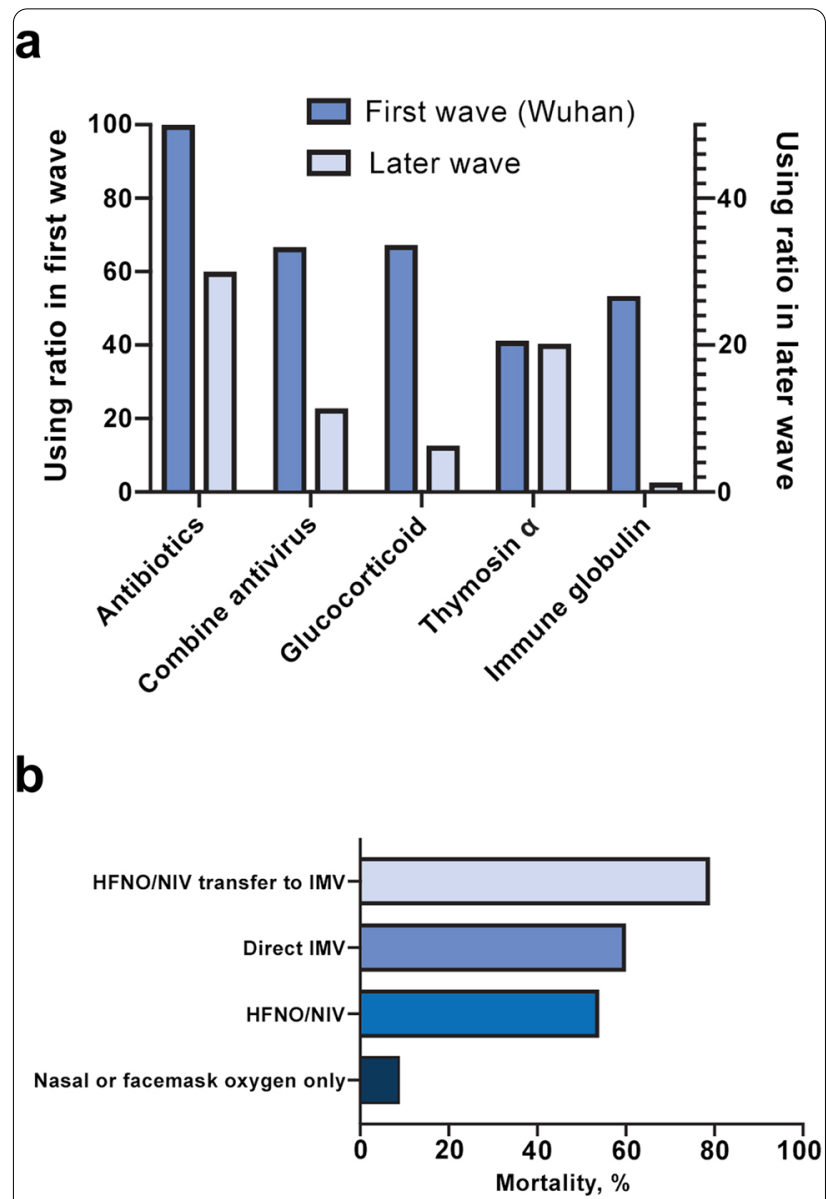

Fig. 1 a The ratio of various drugs used in critically ill patients with COVID-19 in the first and later wave. Data of the first wave were from our previous study (Intensive Care Med 2020 46:1863-1872) and data of later wave were collected in the later outbreak of COVID-19 in Xinjiang and Heilongjiang provinces. $\mathbf{b}$ The mortality of the critically ill patients with COVID-19 who received different ventilatory support. HFNO high flow nasal oxygen; NIV non-invasive ventilation; IMV invasive mechanical ventilation. These data are from our previous study (Lancet Digit Health 2021; 3: e166-74) 
learning approach to identify inflammatory phenotypes and found that glucocorticoids were associated with lower mortality in patients with a highly inflammatory phenotype [10].

Third, the shunt is an extreme form of V/Q mismatching. In most ARDS cases, V/Q mismatching prevails over the shunt. The prone position can recruit collapsed alveoli in gravity-dependent regions and reduce pulmonary shunting in patients with ARDS. Sun et al. first recommended awake prone position ventilation in patients with severe COVID-19 [11]. Later studies showed that awake prone position ventilation could significantly improve oxygenation but not avoid the rate of intubation [12]. We found that prone positioning substantially improved overall V/Q matching by decreasing dead space; however, this effect was lost after reverting to the supine position in patients with COVID-19. A prolonged prone position is associated with better outcomes. Most of our patients tolerated more than $12 \mathrm{~h}$ or longer in the prone position every day. In the critical care expert consensus, we recommend that spontaneously breathing patients with COVID-19 who need oxygen therapy or have rapid deterioration in chest images should assume a prone position for at least $12 \mathrm{~h}$ per day [13].

Fourth, high-flow nasal oxygen (HFNO) therapy is commonly used to improve hypoxemia in clinical practice. However, delayed intubation will lead to higher mortality in patients who receive HFNO therapy. We found that intubated patients after HFNO or noninvasive ventilation (NIV) failure had significantly higher mortality than patients who received directed invasive mechanical ventilation (Fig. 1b). It is difficult to assess the respiratory drive during HFNO therapy. However, under a certain pressure support level during NIV, a higher tidal volume reflects higher respiratory effort. Every patient who received HFNO therapy should switch to NIV for a short time. We performed an expiratory occlusion maintained for the duration of a single breath during NIV to measure expiratory occlusive pressure (NIV test), which also reflects respiratory effort. However, during NIV, occlusion pressure may underestimate inspiratory driving. Chinese expert consensus suggested that when the tidal volume was continuously greater than $9-10 \mathrm{ml} / \mathrm{kg} \mathrm{PBW}$, patients need intubation and should receive lung-protective ventilation [13].

Additionally, we believe that ventilation strategies should be the same as those in ARDS patients with the same respiratory mechanics and clinical characteristics, regardless of whether ARDS is induced by COVID-19. Ferrando et al. analyzed 742 patients with ARDS caused by COVID-19 and found that these patients had similar characteristics to other traditional ARDS patients [5]. SSC guidelines also recommend ventilation strategies similar to those of traditional ARDS for COVID-19 ARDS patients [4]. However, Gattinoni et al. reported that COVID-19 pneumonia is categorized into two phenotypes: type $\mathrm{H}$ and type $\mathrm{L}$ [14]. Chiumello $\mathrm{D}$ et al. used computed tomography scans and found that COVID-19ARDS was characterized overall by higher compliance and lung gas volume for a given $\mathrm{PaO}_{2} / \mathrm{FiO}_{2}$ [15]. We also had some COVID-19 ARDS patients with high respiratory compliance and low lung recruitability. Positive end expiratory pressure (PEEP) settings based on the $\mathrm{FiO}_{2} /$ PEEP table may result in extremely high driving pressure and plateau pressure in these patients.

\section{Personal protection should follow the World Health Organization (WHO) guidelines}

Personal protective equipment is important for the prevention of COVID-19. However, during the first wave of the pandemic, overprotection was common due to fear of infection. Medical staff members wore hats, N95 masks and surgical masks, long-sleeved gowns and coveralls, goggles and face shields and at least two pairs of gloves when he or she entered the isolation ward to take care of COVID-19 patients. A negative pressure headcover was used during suction or other operations. These strategies further aggravate medical and social resource shortages. In addition, excessive protection can cause psychological pressure and affect the quality of medical management. Later, we followed the guidelines from the WHO to use personal protective equipment in various circumstances.

\section{Strategies to be employed after COVID-19 in China}

Although COVID-19 is almost controlled in China, we need to take measures to prevent the recurrence of COVID-19 due to the very serious condition worldwide. In addition, strategies should be implemented to cope with outbreaks, such as COVID-19, in the future. The critical care system should be rebuilt and strengthened. ICU beds should be doubled, and residents should receive critical care training. In addition, general wards are designed such that some beds can be converted into ICU beds when needed. In addition, critical care staff are encouraged to receive vaccination to prevent infection when they take care of COVID-19 patients.

In conclusion, the COVID-19 pandemic has changed the critical care delivery system in China. Critical care resources should be prepared for the next disaster, similar to COVID-19, in the future. Clinical studies are encouraged during the pandemic to identify effective prevention and intervention strategies to control the disease. 


\section{Author details}

1 Jiangsu Provincial Key Laboratory of Critical Care Medicine, Department of Critical Care Medicine, School of Medicine, Zhongda Hospital, Southeast University, Nanjing 210009, China. ${ }^{2}$ State Key Laboratory of Complex, Severe and Rare Diseases, Medical ICU, Peking Union Medical College Hospital, Peking Union Medical College and Chinese Academy of Medical Sciences, Beijing 100730, China.

\section{Funding}

This work was supported, in part, by research grants from the Ministry of Science and Technology of the People's Republic of China (grant numbers: 2020YFC0841300 and 2020YFC0843700), the National Natural Science Foundation of China (grant number: 81930058) and the Chinese Academy of Medical Sciences (CAMS) Innovation Fund for Medical Sciences (CIFMS) from the Chinese Academy of Medical Sciences (2019-12M-1-001 and 2020-12M-2-005).

\section{Declarations}

\section{Conflicts of interest}

All authors report no conflicts of interest to declare.

\section{Publisher's Note}

Springer Nature remains neutral with regard to jurisdictional claims in published maps and institutional affiliations.

\section{Received: 24 March 2021 Accepted: 27 May 2021}

Published online: 22 June 2021

\section{References}

1. Wu ZH, McGoogan JM (2020) Characteristics of and important lessons from the coronavirus disease 2019 (COVID-19) outbreak in China: summary of a report of 72314 cases from the Chinese center for disease control and prevention. JAMA 323:1239-1242. https://doi.org/10.1001/jama.2020.2648

2. Wunsch $H$, Angus DC, Harrison CO, Fowler R, Hoste EAJ, Keizer NF, Kersten A, Linde-Zwirble WT, Sandiumenge A, Rowan KM (2008) Variation in critical care services across North America and West Europe. Crit Care Med 36:2787-2793. https://doi.org/10.1097/ccm.0b013e318186aec8

3. Wang Y, Lu X, Li Y, Chen H, Chen T, Su N, Huang F, Zhou J, Zhang B, Yan F, Wang I (2020) Clinical course and outcomes of 344 intensive care patients with COVID-19. Am J Respir Crit Care Med 201:1430-1434. https://doi.org/ 10.1164/rccm.202003-0736le

4. Alhazzani W, Møller MH, Arabi YM, Loeb M, Gong MN, Fan E, Oczkowski S, Levy MM, Derde L, Dzierba A, Du B, Aboodi M, Wunsch H, Cecconi M, Koh Y, Chertow DS, Maitland K, Alshamsi F, Belley-Cote E, Greco M, Laundy M, Morgan JS, Kesecioglu J, McGeer A, Mermel L, Mammen MJ, Alexander PE, Arrington A, Centofanti JE, Citerio G, Baw B, Memish ZA, Hammond N, Hayden FG, Evans L, Rhodes A (2020) Surviving sepsis campaign: guidelines on the management of critically ill adults with coronavirus disease 2019
(COVID-19). Intensive Care Med 46:854-887. https://doi.org/10.1097/ccm. 0000000000004363

5. Ferrando C, Suarez-Sipmann F, Mellado-Artigas R, Hernández M, Gea A, Arruti E, Aldecoa C, Martínez-Pallí G, Martínez-González MA, Slutsky AS, Villar J, COVID-19 Spanish ICU Network (2020) Clinical features, ventilatory management, and outcome of ARDS caused by COVID-19 are similar to other causes of ARDS. Intensive Care Med 46:2200-2211. https://doi.org/10. 1007/s00134-020-06192-2

6. Xie J, Tong Z, Guan X, Du B, Qiu H, Slutsky AS (2020) Critical care crisis and some recommendations during the COVID-19 epidemic in China. Intensive Care Med 46:837-840. https://doi.org/10.1007/s00134-020-05979-7

7. Tobin MJ, Laghi F, Jubran A (2020) Why COVID-19 silent hypoxemia is baffling to physicians. Am J Respir Crit Care Med 202:356-360. https://doi.org/ 10.1164/rccm.202006-2157cp

8. Guillon A, Hiemstra PS, Si-Tahar M (2020) Pulmonary immune responses against SARS-CoV-2 infection: harmful or not? Intensive Care Med 46(10):1897-1900. https://doi.org/10.1007/s00134-020-06170-8

9. RECOVERY Collaborative Group, Horby P, Lim WS, Emberson JR, Mafham M, Bell JL, Linsell L, Staplin N, Brightling C, Ustianowski A, Elmahi E, Prudon B, Green C, Felton T, Chadwick D, Rege K, Fegan C, Chappell LC, Faust SN, Jaki T, Jeffery K, Montgomery A, Rowan K, Juszczak E, Baillie JK, Haynes R, Landray MJ (2021) Dexamethasone in hospitalized patients with Covid-19. New Engl J Med 384:693-704. https://doi.org/10.1056/nejmoa2021436

10. Chen H, Xie J, Su N, Wang J, Sun Q, Li S, Jin J, Zhou J, Mo M, Wei Y, Chao Y, Hu W, Du B, Qiu H (2020) Corticosteroid therapy is associated with improved outcome in critically ill coronavirus disease 2019 patients with hyperinflammatory phenotype. Chest 3692:35352-35356. https://doi.org/10.1016/j. chest.2020.11.050

11. Sun $Q$, Qiu H, Huang M, Yang Y (2020) Lower mortality of COVID-19 by early recognition and intervention: experience from Jiangsu Province. Ann Intensive Care 10:33. https://doi.org/10.1186/s13613-020-00650-2

12. Coppo A, Bellani G, Winterton D, Di Pierro M, Soria A, Faverio P, Cairo M, Mori S, Messinesi G, Contro E, Bonfanti P, Benini A, Valsecchi MG, Antolini L, Foti G (2020) Feasibility and physiologic effects of prone positioning in non-intubated patients with acute respiratory failure due to COVID-19 (PRON-COVID): a prospective cohort study. Lancet Respir Med 8:765-774. https://doi.org/10.1016/s2213-2600(20)30268-x

13. Diagnosis and management protocol of COVID-91 in China. http://www. nhc.gov.cn/xcs/zhengcwj/202104/7de0b3837c8b4606a0594aeb0105232b. shtml. Accessed 20 May 2021

14. Gattinoni L, Chiumello D, Caironi P, Busana M, Romitti F, Brazzi L (2020) Camporota L (2020) COVID-19 pneumonia: different respiratory treatments for different phenotypes? Intensive Care Med 46:1099-1102. https://doi. org/10.1007/s00134-020-06033-2

15. Chiumello D, Busana M, Coppola S, Romitti F, Formenti P, Bonifazi M, Pozzi T, Palumbo MM, Cressoni M, Herrmann P, Meissner K, Quintel M, Camporota L, Marini JJ, Gattinoni L (2020) Physiological and quantitative CT-scan characterization of COVID-19 and typical ARDS: a matched cohort study. Intensive Care Med 46:2187-2196. https://doi.org/10.1007/s00134-020-06281-2 